
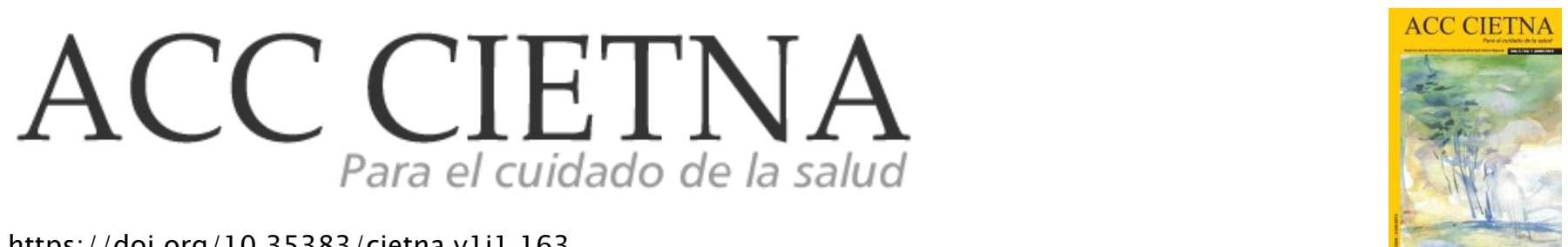

https://doi.org/10.35383/cietna.v1i1.163

\title{
Rol fundamental del adulto mayor en la familia extensa: crianza de los nietos
}

\author{
Mechan Salazar Romi ${ }^{1}$, Díaz Manchay Rosa Jeuna ${ }^{2}$
}

\begin{tabular}{ll} 
INFORMACIÓN DEL ARTíCULO & RESUMEN \\
\hline Historia del artículo: & La presente investigación cualitativa etnográfica tuvo como objetivo: \\
Recibido el 10 de mayo de 2013 & describir, analizar y comprender el rol fundamental del adulto mayor: \\
Aceptado el 5 de noviembre de 2013 & crianza de los nietos. El marco teórico lo constituyó Bustamante (2004), \\
& Forttes (2004), Soto y Darío (1996) y Gámez (2002) para fundamentar \\
\hline Palabras claves: & rol, adulto mayor y familia, los abuelos y la crianza, alternativas al uso \\
Rol & de castigo. En la recolección de los datos se utilizó la observación \\
Adulto mayor & participante y la entrevista etnográfica en seis familias extensas, \\
& habiendo participado en su mayoría, todos los integrantes de la familia \\
& incluyendo el adulto mayor. Fueron considerados los principios de la \\
& Bioética personalista y los criterios de rigor científico. Como \\
& consideración final tenemos que el adulto mayor desempeña diferentes \\
& roles cuando vive dentro de una familia extensa, porque aportan \\
creencias culturales, conocimientos, experiencias y enseñanzas en el & \\
cuidado y en la crianza de los nietos.
\end{tabular}

The fundamental role of the older adult in the extended family: raising the grandchildren

\section{ABSTRACT}

Keywords:

Role

Senior

Extended family

This ethnographic qualitative research, aimed to describe, analyze and understand the fundamental role of the elderly: raising grandchildren. The theoretical framework was constituted by Bustamante (2004), Forttes (2004), Soto and Darío (year) and Gámez (2002) to support role, elderly and family, grandparents and parenting, alternatives to punishment in collecting data is used participant observation and

\footnotetext{
1 Licenciada en Enfermería. Enfermera del Centro de Salud de Piura, Piura, Perú. Email: rmechan@hotmail.com

2 Doctora en Ciencias de Enfermería. Directora de Escuela de Enfermería de la Universidad Católica Santo Toribio de Mogrovejo. Chiclayo- Perú. 
ethnographic interviews in six extended families, having participated in most cases, all family members including the elderly. Were considered the principles of bioethics personality and scientific rigor criteria. Obtaining as final consideration the different roles played by older adults when they live within an extended family because they bring cultural beliefs, knowledge, experiences and lessons in the care and upbringing of their grandchildren.

\section{Introducción}

Los adultos mayores perpetúan las tradiciones culturales de la humanidad, trasmitiéndolas de generación en generación. A nivel mundial, hay un incremento acelerado de la población adulta mayor, para el año 2050 los mayores de 65 años representarán el $21 \%$ de la población del mundo; en el Perú, este grupo poblacional representa el $7,6 \%$ de la población total ${ }^{1}$. Según estadísticas en Latinoamérica, una de cuatro familias tiene una persona mayor entre sus miembros; en Chile casi el $30 \%$ de los hogares tiene un adulto mayor, cuya función principal es la crianza de los nietos². En nuestro país el 2,4 millones de adultos mayores son responsables de criar a los nietos, por el ausentismo de los padres; y el $8,80 \%$ de la población adulta mayor encabeza una familia extensa ${ }^{3,4}$.

En las sociedades tradicionales, el abuelo y la abuela son el tronco de la familia, representan la sabiduría, el buen consejo, el respeto y la identidad familiar ${ }^{5}$. Los adultos mayores viven muchas veces en compañía de sus hijos y nietos formando la familia extensa6. Esta situación es muy común de observar en la cultura peruana, ya que la actual situación socioeconómica hace que el vivir en familia sea una necesidad, más que un aspecto cultural. Específicamente, en la ciudad de Monsefú, las investigadoras han podido observar que los adultos mayores tienen generalmente la costumbre de vivir con sus hijos y nietos; esto porque las hijas son madres solteras, los yernos 0 hijos ganan muy poco, y otros porque mantienen lazos afectivos muy estrechos, y creen que nadie mejor que los abuelos para cuidar y educar a los nietos.

Con ello, se puede rescatar que el adulto mayor también participa y colabora cuidando a su familia, ocupando nuevos roles familiares y transmitiendo sus patrones culturales acerca de la crianza y medidas de corrección de los niños, la preparación de los alimentos, preparación de remedios caseros para cuidar la salud y curar las enfermedades, entre otros aspectos. De esta manera, los adultos mayores que viven en la familia extensa, cumplen ciertos roles como llevar a los nietos al colegio o recogerlos, hacer que cumplan con las tareas del colegio, verlos que no salgan a la calle, y para curar a los miembros de la familia utilizan plantas medicinales o acuden al curioso o curandero.

El uso de plantas medicinales, a veces la negativa de que acudan al centro de salud para que vacunen a los primero hay que llevarlo al curioso para que le niños o les apliquen inyectables, la creencia de que "pasen" el cuy, el alumbre, la vela, el periódico, le saquen chucaque, lo santigüen, etc. Al parecer el cuidado sería recíproco, se necesitan, complementan y apoyan, rescatando el rol actual que cumple el adulto mayor cuando vive y convive en una extensa formada por sus hijos (as), nueras, yernos y nietos(as).

Frente a lo descrito, se formula la siguiente pregunta de investigación: ¿Cómo el adulto mayor desempeña familia extensa del distrito de Monsefú, 2011? Se el rol de crianza de los nietos cuando vive en la y analizar el rol del adulto mayor 
en la crianza de los tuvo los siguientes objetivos: Describir, comprender nietos al vivir con la familia extensa en el distrito de Monsefú, 2011. Esta investigación refleja y rescata uno de los roles que desempeña el adulto mayor dentro de la familia extensa que es la crianza de los nietos, quien merece consideración, respeto y ser tratado como una persona útil, que colabora y apoya en el desarrollo de los roles familiares; pero desvalorizando al adulto mayor; aspectos que deben actualmente la sociedad y las familias están ser considerados cuando la enfermera cuida al adulto mayor y a la familia, en cualquier ámbito donde desarrolle su actuar.

\section{Metodología}

La presente investigación es de tipo cualitativa con enfoque etnográfico como tal procura lograr la descripción holística e intenta analizar exhaustivamente, la realidad en su contexto natural, intentando interpretar los fenómenos de acuerdo al significado que tienen para las personas implicadas. ${ }^{7}$

La etnografía es un proceso sistematizado de observar, describir, detallar, documentar y analizar los patrones propios de una determinada cultura; se traduce etimológicamente como "el estudio de las etnias" y significa el análisis de modo de vida de una raza o grupos de individuos, mediante la observación y descripción de lo que la gente hace, cómo se comportan e interactúan entre sí, para describir creencias, valores, motivaciones, perspectivas y cómo estos pueden variar en diferentes momentos y circunstancias, es decir, que describe las múltiples formas de vida de los seres humanos.8,9 Teniendo en cuenta esto, la investigación etnográfica permitió a las investigadoras observar cómo se desenvuelve el adulto mayor cuando vive con su familia extensa y de qué manera desempeña el rol de crianza de los nietos, para así establecer sus relaciones, costumbres, prácticas culturales, creencias, comportamientos, etc. en su contexto cultural, la ciudad de Monsefú.
El escenario es el emplazamiento donde el investigador se va a situar como observador, que puede ser una comunidad (una aldea o pueblo, un barrio, un centro de salud, la sala de un hospital, entre otros) o un grupo (los ancianos de una residencia, un colectivo profesional, los alumnos de un colegio, entre otros). ${ }^{10} \mathrm{La}$ investigación se llevó a cabo en los hogares de los adultos mayores que viven con su familia extensa en el distrito de Monsefú. Este distrito es uno de los pueblos Lambayecanos con profundo y brillante pasado histórico. Su área territorial abarca $44,94 \mathrm{~km}^{2}$ y tiene una población de 30.591 habitantes (estimación año 2000 - fuente INEI). ${ }^{11}$ Uno de sus atractivos costumbristas es la celebración de la Feria Patronal de Jesús Nazareno Cautivo, considerado el patrón del pueblo; otra fiesta importante es el Fexticum, fiesta popular más importante del departamento de Lambayeque, en la que se expone las costumbres, la creación cultural gastronómica e intelectual del norte del Perú Muchick. Creen en el curanderismo y utilizan el huevo, velas, periódicos, para el mal de ojo o el chucaque.

Formaron parte del Grupo Cultural todas las personas adultas mayores y sus familias extensas, que residieron en el distrito de Monsefú, que reunieron los siguientes criterios de inclusión: Familias extensas que estén compuestas por personas adultas mayores de 65 años y que vivan con los hijos, nueras, yernos, nietos, en el mismo hogar. No se incluyeron a las familias que tienen adultos mayores que padezcan de enfermedades crónicas - degenerativas y alteraciones mentales. Los informantes estuvieron constituidos por las personas adultos mayores $y$ sus familiares mayores de 18 años, que cumplieron con los criterios de inclusión y exclusión antes mencionados, fueron seis familias extensas y se determinó por la técnica de saturación, es decir, cuando la ocurrencia de las descripciones se repetía o los informantes del estudio no aportaron nuevos datos a la investigación. 
Antes de la recolección de los datos se procedió a realizar la validación de la entrevista la misma que se realizó con dos adultos mayores y sus respectivas familias extensas del distrito de Monsefú; estas personas no formaron parte de los informantes y de acuerdo a los resultados permitió modificar, anular o incrementar algunas preguntas para su mejor comprensión. Después que las familias aceptaron participar, firmaron el consentimiento informado y las técnicas e instrumentos de recolección de datos: la se quedó en la hora y fechas de las visitas, para aplicar observación participante, la entrevista etnográfica y el diario de campo. Las visitas duraron aproximadamente entre tres a cuatro horas diarias, y se realizaron un promedio de siete visitas por familia, en el lapso de tres meses, desde abril hasta junio de 2010.

Para procesar y analizar los datos, se utilizaron las cuatro fases del análisis temático: identificación y caracterización de los descriptores y sus componentes; categorización de los elementos identificados y clasificados en categorías; identificación de los patrones; temas relevantes, formulaciones teóricas y recomendaciones. ${ }^{12}$

El análisis de los datos que el investigador alcanza recolectando, describiendo y documentando la materia prima y los datos brutos cuando inicia el análisis de los datos relacionados al tema, objetivo, y cuestiones del estudio. Se tuvieron en cuenta los principios de la Bioética personalista: principio de respeto a la vida humana, principio de libertad y responsabilidad, principio de sociabilidad y subsidiaridad. ${ }^{13}$ Durante la recolección de los datos se ayudó y educó a las familias, en algunas prácticas culturales inadecuadas que podían dañar su salud.

La investigación cualitativa se basa en los siguientes criterios de rigor científico: la credibilidad, la transferibilidad y la confirmabilidad, aspectos que tienen como fin aumentar la calidad de la información. ${ }^{14}$ Principio de credibilidad es la responsabilidad del investigador al recaudar los datos fidedignos, mediante comprobaciones. Se logró a través del uso de la observación, el chequeo con los informantes, haciendo que los informantes revisen la trascripción de las entrevistas. El principio de transferibilidad se refiere fundamentalmente a la posibilidad de generalizar los resultados a la población de la cual se ha estudiado una parte, o hacer ciertas inferencias lógicas sobre situaciones o poblaciones que sean muy similares a la estudiada. El principio de confirmabilidad consiste en la comprobación por parte de un investigador externo de la adecuación de la estrategia seguida, la calidad de la información recolectada, la coherencia interna de los datos, la relación entre estos y las interpretaciones realizadas.

\section{Resultados, análisis y discusión}

En la presente investigación, las familias tenían el abuelo(a) "ideal", adultos mayores, que cumplen diversos roles con cariño y amor, en especial la crianza de los nietos, que incluye prepararle su alimentación, llevarlos al colegio, jugar, ayudar con las tareas, lavar su ropa, y velar por su formación personal, por ello corrigen, castigan y ponen las reglas, entre otros cuidados; aunque no reciban sueldo, dan todo lo que tienen a sus nietos e hijos. Los adultos mayores que son abuelos sienten una gran satisfacción cuando comparten el cuidado de sus nietos, además de hacerlos sentir útiles y activos. Esta colaboración repercute positivamente, tanto en ellos como en los niños; es una experiencia muy enriquecedora para ambos, se forma un fuerte lazo afectivo entre abuelo (a) nieto (a). La participación de los abuelos al cuidar a los niños puede ayudar a mejorar o mantener una adecuada relación conyugal, ya que esta podrá disponer de momentos para ellos mismos, sin la presencia de los hijos; pero que esto no signifique descuidar a los hijos y dejar solos a los adultos mayores en la responsabilidad de criar a los niños.

En el distrito de Monsefú los abuelos prestan una importante ayuda dentro de la familia, cuidando de los niños mientras los padres están ausentes. Los abuelos tienen una función importante en el 
desarrollo de los niños; transmiten experiencias e inculcan valores, no solo por sus historias, sino también como ejemplo de sabiduría y vida. Ellos deben tomar en cuenta que una adecuada relación entre abuelos y padres mejora incluso la relación de padres e hijos, y fortalece ese núcleo de amor, ayuda en la formación de niños y niñas con buena autoestima, autonomía, creatividad, solidaridad y salud, es decir, mejores personas.

“...mi madre es atenta, es como si fuera su segunda madre, porque lo ha visto desde que nació y le inculca buenos valores...", “...la forma de corregir de mi padre es levantando la voz, pero mi madre es de carácter más fuerte que mi padre y a veces les jala las orejas".

Según los discursos, los abuelos tratan de corregir a los nietos, levantando la voz, a veces castigando físicamente, inculcan la obediencia, respeto $y$ responsabilidad para cumplir sus obligaciones. Al parecer para ellos una forma de crianza es el castigo físico ya que así han educado a sus hijos y así ellos fueron educados y consideran que por eso son buenas personas, e incluso critican la forma de corregir actualmente ahora a los hijos.

"...Cuando desobedecen mis nietos, les jalo las orejas, o los grito...cuando a veces no cumplen con las tareas del colegio los castigo con la correa aunque se enoje mi hija... yo he criado así a mis hijos $y$ son buenas personas, por eso sigo corrigiéndoles así a mis nietos... porque si uno no le pone la mano fuerte más adelante van a querer pegarnos...pero mi esposo dice mejor es hablarles antes que pegarles...."

Los adultos mayores pueden prestar una valiosa ayuda dentro del ámbito familiar, funcionando como un apoyo para sus hijos (para el padre principiante es una valiosa fuente de información); pero, sobre todo, tienden a convertirse en educadores y hasta cómplices de los nietos, quienes regularmente perciben a los abuelos como sabios. Los abuelos tampoco pueden remplazar a los padres, porque daría por resultado que las personas adultas mayores tendrían la responsabilidad absoluta de criar a sus nietos, lo que conlleva a consecuencias como estrés, cansancio, etc., siendo recomendable que los hijos no deleguen sus responsabilidades a terceros y sean conscientes de las limitaciones cuando deseen pedir ayuda a los abuelos.

Además de lo mencionado, las investigadoras observaron que los abuelos utilizan diversas creencias y objetos comúnmente empleadas para sanar algunas enfermedades populares.

"...la creencia que tenemos que antes de ir al doctor, primero nos limpiarnos con un cuy, es una manera de decir que el cuy recoge nuestro dolor o malestar...", "El alumbre es contra el susto...el periódico se pasa por todo el cuerpo y luego se quema, si suena como cancha es viento, puede ser que un perro callejero lo asustó... el huevo se pasa por todo el cuerpo, se quiebra en medio vaso de agua y sale como una cosa blanca, es que tiene viento...", "...el chucaque se quiebra con tierrita, la tierrita se agarra del rincón de la casa pero tiene que estar bien molida se soba la barriga y luego le da un poco de yonque con pimienta...".

Los abuelos antes de acudir al centro de salud, limpian con cuy al familiar que padece alguna enfermedad, esta es una creencia de los abuelos que es trasmitida de generación en generación. Otras de las creencias de los adultos mayores es el alumbre contra el susto la cual se utilizan en niños, para prevenir el mal comportamiento que adquiere cuando un perro callejero los asusta, entre otros. En esta versión nos hablan sobre las formas de quebrantar el chucaque al parecer según la pimienta y el yonque es una forma peligrosa ya que puede dañar el estómago, su combinación que se realiza siente una quemadura dentro del estómago, a diferencia del cuy es utilizado en la cultura andina para restituir la salud.

Asimismo, el adulto mayor usa las plantas medicinales para curar enfermedades: estas creencias o prácticas son heredadas de sus 
ancestros. Actualmente nos encontramos con personas que al no encontrar solución en la medicina convencional se ven en la necesidad de recurrir a prácticas que les dejaron sus antepasados por ser más factibles y haber dado resultados positivos.

"Yo me cuido con medicina natural como es el eucalipto que es contra la gripe y el dolor del riñón, el matico, la cola de caballo, el orégano contra los cólicos menstruales y el llantén... pero otras personas compran pastillas en la farmacia..."

Estas personas recurren a lo natural, ya que tomando pastillas puede que les afecte su salud y ellos saldrán perjudicados por los gastos y además porque no cuentan con los recursos necesarios para ir al centro de salud, ya que además está lejos de sus casas. Estas personas tienen otras creencias por las cuales son reemplazadas por las llamadas "pajitas" y logrando así su cura ante cualquier enfermedad.

Actualmente hay una tendencia a que se formen familias extensas, las que están conformadas por abuelos, hijos, nietos, nueras o yernos y viven en el mismo hogar, existe ayuda mutua entre sus integrantes cumpliendo diversos roles $y$ responsabilidades. Sin embargo, en la familia extensa se puede presentar conflictos por la forma peculiar que tienen los abuelos al emplear medidas correctivas o la disciplina, que pueden ser criticadas o acertadas en la actualidad. El abuelo (a) es quien elabora las reglas del hogar, puesto que ellos son en su mayoría los dueños de la casa; además cuidan la salud de la familia, usando sus rituales y creencias populares aprendidas $y$ trasmitidas de generación en generación.

Una familia extensa tiene sus ventajas y problemas, aunque tener los hijos es cosa de cada pareja, implica al resto de la familia, en especial a los abuelos, por ello hay que delimitar los roles. ${ }^{15}$ La vida está enmarcada en una red de derechos y obligaciones llamadas relaciones de roles; los roles familiares son críticos y centrales, un individuo debe aprender a desempeñar exitosamente, no solo para un funcionamiento adecuado de rol sino también para el éxito de la familia. ${ }^{16}$

Para el adulto mayor vivir en familia extensa es desempeñar diferentes roles, significa reciprocidad, pues reciben ayuda de su familia y también ayudan a cuidarla, en su mayoría se sienten reconocidos, queridos y útiles, sobre todo, por lo nietos.

En nuestra sociedad, es imprescindible la ayuda de otras personas para cuidar a los hijos, es muy difícil encontrar a alguien "de confianza" que permita tener la certeza de que los hijos van a estar bien cuidados, y para esto se recurre a la ayuda de los abuelos. De esta forma, los padres se sienten más tranquilos y tienen la seguridad de que sus hijos reciben los mejores cuidados, cariño y afecto. Además, con los abuelos los nietos crecen en un entorno familiar lleno de cariño, lo que aporta grandes beneficios a los pequeños que se sienten protegidos y queridos. Los abuelos sienten una gran satisfacción cuando comparten el cuidado de sus nietos, además de hacerlos sentir útiles y activos.

Los abuelos tienen una importante labor en la crianza de los nietos, tienen la función de transmitir conocimientos generacionales del pasado, pueden escuchar, comprender y sostener a sus nietos, cuando sus padres no pueden hacerlo; hablan de la historia familiar, de las tradiciones, de cómo eran sus padres cuando niños, etc. 17 El abuelo ideal está disponible y es cariñoso, cercano al nieto, acepta el ser abuelo como un aspecto más de su identidad personal, familiar y social. ${ }^{18}$ Sin embargo, existe el "síndrome del abuelo esclavo", que es un trastorno que afecta a personas mayores obligadas a cuidar de sus nietos, asumiendo el rol de padres $y$ dejando de lado su propia vida. ${ }^{19}$

En la convivencia diaria del adulto mayor con la familia extensa, cada miembro cumple diferentes roles, lo asumen de acuerdo a las necesidades que 
se van presentando en la familia; por ejemplo el adulto mayor hace las compras del mercado, prepara a los nietos para el colegio, los recoge del colegio si son pequeños, pero si son adolescentes están pendientes de que ellos regresen a casa, asimismo les preparan los alimentos mientras que los padres de los niños regresan del trabajo o de otras responsabilidad fuera de su hogar.

La puericultura es el arte de cultivar a los niños, es el arte de la crianza, incluye el instruir, educar y dirigir; los protagonistas son principalmente el padre y la madre, pero por diferentes circunstancias, terminan participando los abuelos.20 Es importante establecer acuerdos entre los padres y los abuelos relacionado con la crianza de los hijos, como es el establecimiento de normas y la educación en general. La ayuda de los abuelos debe ser totalmente voluntaria, y tener en cuenta su edad y estado de salud física y emocional. ${ }^{21}$

En la presente investigación, los abuelos tratan de corregir a los nietos, levantando la voz, a veces los castigan físicamente, inculcándoles a obedecer, respetar y, sobre todo, a cumplir con sus obligaciones. Al parecer para ellos una forma de crianza es el castigo físico ya que así han educado a sus hijos y así ellos fueron criados, y que por eso son buenas personas, que no hacen daño a los demás y que valoran a su familia.

Una de las formas de corregir son los palmazos y jalones de orejas para que los niños obedezcan.

Actualmente se recomienda a los padres el empleo de castigos moderados, no físicos como la retirada de un permiso o privilegio durante un periodo de tiempo (por ejemplo, quedarse sin salir de casa, sin ver la televisión, sin su juguete favorito, etc.). Tan importante como corregir el comportamiento inadecuado, es recompensar lo que el niño hace bien. Las recompensas de tipo social como el elogio y la aprobación verbal, así como las expresiones no verbales (por ejemplo, una sonrisa o un gesto) o el contacto físico son formas muy efectivas para enseñar al niño a comportarse adecuadamente. 22,23

El adulto mayor desempeña diferentes roles cuando vive dentro de una familia extensa, porque aportan creencias culturales, conocimientos, experiencias y enseñanzas como cuidador de la salud familiar, cuidan a los nietos, realizan los quehaceres del hogar, muchas veces apoyan para el gasto familiar. Sin embargo, hay conflictos familiares con hijos, nueras, yernos, cuando tratan de corregir a sus nietos o utilizan rituales extraños para curar.

El adulto mayor posee ciertas creencias, rituales y tradiciones culturales que las practican en su vida cotidiana, las cuales han sido aprendidas de sus antepasados y son trasmitidas a las nuevas generaciones, entre ellas tenemos: remedios caseros, el uso de ciertos objetos y animales para curar del susto, el ojo y el chucaque. Los niños son quienes mayormente sufren del "susto" para ello los abuelos recurren a sus prácticas usuales como pasarles el cuy por el cuerpo, limpiarlos con cuy, vela o alumbre. Los adultos mayores en primera instancia recurren a estas prácticas, ya sea limpiar con vela, cuy, etc. y prefieren hacerlo antes de Ilevarlo al centro de salud, muchas veces por haber escuchado o tener experiencias negativas. Los abuelos son muy creyentes del el ojo o chucaque, el ojo solo les da a los más pequeños de casa, por ser inocentes y sensibles al mal humor de los adultos, en cambio el chucaque se da mayormente en personas adultas. Estas personas se encuentran muy aferradas a sus creencias porque han comprado la efectividad de dichos tratamientos. Aspectos que deben ser considerados en el cuidado del personal de salud y además investigados a profundidad, para fomentar la interculturalidad y la inclusión social.

En el distrito de Monsefú los abuelos prestan una importante ayuda dentro de la familia, cuidando de los niños mientras los padres están ausentes. Los abuelos tienen una función importante en el desarrollo de los niños; transmiten experiencias e 
inculcan valores, no solo por sus historias, sino también como ejemplo de sabiduría y vida. Ellos deben tomar en cuenta que una adecuada relación entre abuelos y padres mejora incluso la relación de padres e hijos, y fortalece ese núcleo de amor, es abuelos en la crianza de los niños ayudará a formar decir, un buen trabajo en equipo tanto de padres como niños y niñas con buena autoestima, autonomía, creatividad, solidaridad y salud, es decir, mejores personas.

\section{Referencias bibliográficas}

1. Estimación año 2000 - fuente INEI. Disponible en: http://es.wikipedia.org/ wiki/Distrito_de_ Monsef\%C3\%BA. 2009 junio 01 . Yaipen R. Tradiciones y Costumbres. Disponible en:http: / / munimonsefu.com $/$ fraim. php?enlace $=$ tr adiciones. Consultada el 2009 noviembre 03.

2. Censo de EE.UU. Tabla DP-2. Perfil Seleccionado Características Sociales: "Para los abuelos y la familia que esta criando nietos, 2007. Disponible en: http://www.grandfactsheets.org/ doc/MA-ES07final.pdf. Consultada el 2008 Octubre 5.

3. Censo Lambayeque_2007-Rascacielos del Perú. Disponible en: Inca Skyscrapers - Inkascrapers SkyscraperPeru.htm. Consultada el 2008 Diciembre 01.

4. Varela L. Perfil del adulto mayor Perú - Intra II "Desarrollando repuestas integradas de los sistemas de salud del cuidado de la salud para una población en rápido envejecimiento Intra II -Perú", 2004. Disponible en: http:// www. upch.edu.pe /vrinve/gerontologia/pdfs/prueba_intrall.pdf. Consultada el 2008 Noviembre 17.

5. Importancia de los abuelos en la familia. Disponible en: vidaok.com/importancia-abuelosfamilia.html. Consultada el 23 julio del 2011.

6. Morales M. Chile envejece: Prospectiva de los impactos políticos y sociales de este fenómeno hacia el bicentenario. Disponible en: http:/ / www.observa.uchile.cl/morales.pdf. Consultada el 2009 noviembre 03.

7. Denzin y Lincoln, citado por Rodríguez Gómez Gregorio, en Metodología de la investigación cualitativa. Málaga. Ediciones Aljibe S. L; 1996.

8. Polit F, Hungler P. Investigación Científica en Ciencias de la Salud. México DF: Mc Graw - Hill Interamericana; 2000.

9. Martínez, Miguélez. Ciencia y arte en la metodología cualitativa. México: Editorial Trillas; 2004.

10. Amescua M. 2000. El trabajo de campo etnográficoen salud: Una aproximación a la Observación Participante. Disponible en: < htp://www.index-f/.com/cuali/oservacion. Pdf $>$. Consultada el 2009 junio 03.

11. Exposición de periódicos costumbristas: Municipalidad de Monsefú. Disponible en: munimon se fu.gob.pe/.../exposicion-deperiodicoscostumbr...consultada el 04 mayo del 2009.

12. Spradley, citado por Pezo: A trayectoria percorrida com a depressáo: Estudo Transcultural das vivencias de famílias de Brasil e do Perú. Tese (doutorado en enfermagem) - Escola de Enfermagem da Universidade de Sào Paulo; 2003. 1997.

13. Sgreccia E. Manual de Bioetica. La bioética y sus principios. $4^{\circ}$ Edición. Madrid: Talisio, 2007.

14. Tello C. Gutiérrez, N. Pérez, C. Métodos y técnicas de análisis cualitativo. Sección de Postgrado en Enfermería-Escuela de PostgradoUniversidad Nacional de Trujillo. Trujillo; 2009

15. García M. "Reflexiones sobre la Familia", 1 Abril 1994. Disponible en: .. 
16. Bustamante S. "Enfermería Familia: principios de cuidado a partir del saber (in) común de las familias". Departamento de salud familiar y comunitaria. Facultad de enfermería. Universidad Nacional de Trujillo-2004.

17. Long B, Phipps W. Tratado de Enfermería Médico Quirúrgico: Un enfoque del proceso de Enfermería. Volumen II. Harcourt Brace. Madrid.1999.

18. Forttes B. Alicia: El adulto mayor y la familia. Diplomado de Gerontología a distancia. Programa para el Adulto mayor. 2004.

19. Eduy J. Gerontología: Adulto mayor y familia. "El rol del adulto mayor en la familia". Disponible en:

http://www.buenastareas.com/ensayos/AdultoMa yor-YFamilia/1122864.html.Consultada el 2010 noviembre 06 .

20. Aparicio T. Psicóloga escolar. "Como corregir a sus niños".

Disponible

en: http: / / proyectoaltar.com/2009/12/20/\%C2\%BFu e-dice-labiblia-acerca-de-la-disciplina-de-loshijos/. Consultada el 2010 noviembre 03.

21. Soto G, Darío B: "Los abuelos y la crianza". Disponible en: http://www.buenastareas.com/articulos/los abuelos y la crianza/1122864.html.Consultada 2010 noviembre 06 .

22. Puleva Salud: "El papel del mayor en la sociedad" - Disponible en: http://gerontovital.com/index.php?option =com_c ontent\&view $=$ article\&id $=81$ : familiaadultomayor $\& c$ atid $=34$ : articulos\&ltemid $=53$. Consultada 2010 noviembre 06.

23. Gámez M, Carboles J. y Almendros, C: "Alternativas al uso de castigo" Disponible en: http:/ / www.padresycolegios.com/noticia/2560/L A-OPINI\%C3\%93N-DEL-EXPERTO/funcionacachete-alternativas-uso castigof \%C $3 \% 83 \%$ C2\% ADsico-hijos.html. Consultada el 2010 noviembre 03. 